\title{
Platelet satellitism: unusual cause of spurious thrombocytopenia
}

\author{
Vikrant Singh Bhar ${ }^{1}$ (D) $\cdot$ Rachna Singh ${ }^{1}$
}

Received: 28 June 2019 / Accepted: 17 July 2019 / Published online: 1 August 2019

(C) Springer-Verlag GmbH Germany, part of Springer Nature 2019

\begin{abstract}
Platelet satellitism (PS) is defined as adherence of platelets on the surface of polymorphonuclear leucocytes and imparting a rosette-like appearance especially in ethylenediaminetetraacetic acid (EDTA) whole blood samples. We here present a case of spurious thrombocytopenia resulting from florid platelet adherence on the surface of neutrophils.
\end{abstract}

Keywords Platelet satellitism · Spurious thrombocytopenia

Platelet satellitism (PS) is an uncommon and unique phenomenon observed on peripheral blood smears. It is defined as adherence of platelets on the surface of polymorphonuclear leucocytes and imparting a rosette-like appearance. This might result in spurious or pseudo-thrombocytopenia on complete blood count $(\mathrm{CBC})$ performed on a hematology analyzers.

We here present a case of a 65 -year-old female who underwent $\mathrm{CBC}$ as part of her work up for surgery for right renal calculi. She presented with history of pain in right flank on and off for 2 years. She was afebrile and her urine culture was sterile. The $\mathrm{CBC}$ done using ethylenediaminetetraacetic acid (EDTA) whole blood sample on hematology analyzer (LH780, Beckman Coulter) revealed a platelet count of $70 \times$ $10^{9} / \mathrm{L}$. The other CBC parameters were within normal limits. The reflex peripheral smear prepared and stained with Leishman stain revealed florid platelet adherence on the sur-

Vikrant Singh Bhar

Vikrantbhar86@gmail.com

Rachna Singh

rachnapmch@gmail.com

1 Department of Haematopathology, Artemis Hospitals, Gurugram 122001, India face of polymorphonuclear leucocytes. The platelet adhesion was not seen on the surface of monocytes (Fig. 1).

The platelet count was repeated using heparin anticoagulated whole blood sample and normal platelet counts were obtained $\left(168 \times 10^{9} / \mathrm{L}\right)$ and no platelet adhesion was noted on peripheral smear.

The PS was first described in 1963 by Field and Macleod and since then, many cases have been reported in the literature [2]. The PS is a predominantly in vitro phenomenon and seen in EDTA anti-coagulated samples. However, it has also been rarely reported in heparin and sodium citrate samples.

The mechanism for PS is not completely understood; however, it has been shown that PS is an in vitro phenomenon caused by EDTA-dependent IgG auto-antibodies (antiplatelet and antineutrophil) present in the patient sera. The primary target antigens reported are glycoprotein IIb/IIIa complex of the platelet membrane and the neutrophil $\mathrm{Fc} \gamma$ receptor III ( $\mathrm{Fc} \gamma \mathrm{RIII}$ ) [1]. These auto-antibodies are naturally present in some individuals and no clear correlation between presence of antibodies and specific clinical condition or use of drugs has been reported in the literature.

The occurrence of this rare phenomenon may result in spurious thrombocytopenia which might result in the unnecessary delay in surgical procedures or unnecessary further tests to investigate thrombocytopenia or rare platelet transfusion in emergency settings. 
Fig. 1 Peripheral blood smear showing platelet satellitism on neutrophils (Leishman stain, $x$ 1000)

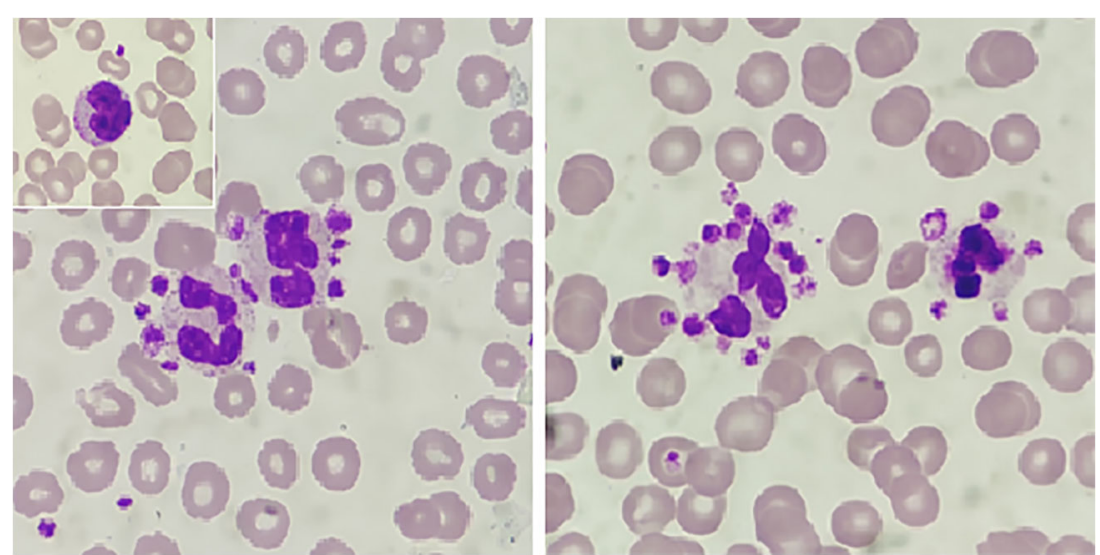

The careful peripheral smear examination to pick PS and repeat $\mathrm{CBC}$ with heparin or citrate anti-coagulated samples will help in obtaining correct platelet counts.

\section{References}

1. Chakrabarti I (2014) Platelet satellitism: a rare, interesting, in vitro phenomenon. Indian J Hematol Blood Transfus. 30(3):213-214
2. Bizzaro N, Goldschmeding R, Von Dem Borne AEGK Platelet satellitism is Fc $\gamma$ RIII (CD 16) receptor-mediated. American Journal of Clinical Pathology 103(6):740-744

Publisher's note Springer Nature remains neutral with regard to jurisdictional claims in published maps and institutional affiliations. 